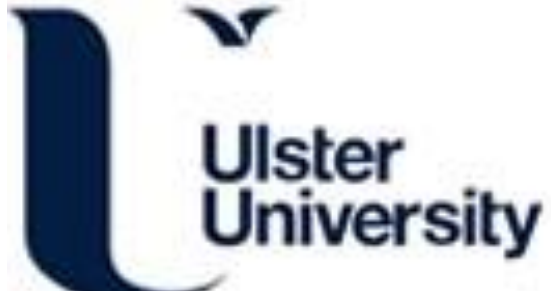

The Role of Context-Aware Computing in Support of People with Dementia

Baumgarten, M., \& Mulvenna, M. (2010). The Role of Context-Aware Computing in Support of People with Dementia. In M. Mulvenna, \& CD. Nugent (Eds.), Supporting People with Dementia Using Pervasive Health Technologies (pp. 131-144). Springer. https://doi.org/10.1007/978-1-84882-551-2

Link to publication record in Ulster University Research Portal

Published in:

Supporting People with Dementia Using Pervasive Health Technologies

Publication Status:

Published (in print/issue): 01/01/2010

DOI:

10.1007/978-1-84882-551-2

Document Version

Publisher's PDF, also known as Version of record

\section{General rights}

Copyright for the publications made accessible via Ulster University's Research Portal is retained by the author(s) and / or other copyright owners and it is a condition of accessing these publications that users recognise and abide by the legal requirements associated with these rights.

\section{Take down policy}

The Research Portal is Ulster University's institutional repository that provides access to Ulster's research outputs. Every effort has been made to ensure that content in the Research Portal does not infringe any person's rights, or applicable UK laws. If you discover content in the Research Portal that you believe breaches copyright or violates any law, please contact pure-support@ulster.ac.uk. 


\title{
Chapter 9 \\ The Role of Context-Aware Computing in Support of People with Dementia
}

\author{
Matthias Baumgarten and Maurice D. Mulvenna
}

\begin{abstract}
There is a strong motivation, in particular in the domain of healthcare, for new perspectives on context-driven research and computing in order to provide next-generation services to people that are tailored to individual needs rather than generalised assumptions that could potentially endanger human life. For that, context awareness is a key requirement in order to reach a better understanding of human-centric computing systems and environments, and subsequently, the deployment of dedicated services that are specifically adapted to the context to which they are applied. Such context-driven services would be able to provide the means of delivering situation-aware and person-centric services that ultimately may even anticipate future behaviour and problems of the user itself and the context in which the user finds themselves. However, the perpetual provision of contextual data in pervasive environments is far from being easy and includes major challenges that vary between environments. The reason for this is not only the sensor diversity within the environments themselves but also the contextual scope to be analysed and the amount of data to be collected and correlated to actually reach a minimum degree of contextual understanding. For that reason, in this chapter, contextual environments have been categorised as well as their interaction into different groups that reflect individual contextual levels of interest of which contextual understanding is required and consequently to which services can be applied.
\end{abstract}

\subsection{Introduction}

On a worldwide context the human population continues to grow rapidly with an increasing percentage represented by the elderly. Considering that the elderly have a higher likelihood of requiring care for various conditions and illnesses, society

\footnotetext{
M. Baumgarten ( $\varangle)$

TRAIL Living Lab, School of Computing and Mathematics, Faculty of Computing and Engineering, University of Ulster, Newtownabbey, Northern Ireland e-mail: m.baumgarten@ulster.ac.uk
} 
is faced with the increasing problem of providing good and cost-effective healthcare on an ever-growing scale. In the UK alone it is estimated that well over a million people will suffer from dementia by 2025 with associated financial cost of over 17 billion pounds (Alzheimers Organisation 2009). Two-third of people with dementia still live in the community and the vast majority of all would like to continue to do so. Thus, there is a general shift from institutional to community care which, however, requires substantial restructuring of healthcare infrastructures and their service provisions. In particular, automated supervision and reinforcement mechanisms could provide the means to enable people in general but in particular people with dementia to remain within their local environment without the constant presence of a human caretaker. The resulting preventative services could help to maintain social interactions, provide assistance and guidance for daily life activities but most importantly they could provide an automated safety network that enables people to remain independent in their own home environment. Nevertheless, in order to provide such services a workable balance has to be found between peoples' needs and the introduction and utilisation of ubiquitous computing environments and the services they facilitate.

Through the deployment of latest sensor technology the move towards "home automation", smart or intelligent environments can be considered to be one that promotes levels of independence and increases personal autonomy (Helal et al. 2003) by being able to deliver services in a context and situation-aware manner.

Within this chapter the role of context-aware computing can play in supporting independent living is evaluated. The chapter also explores how it can be used to improve the quality of life for various aspects. In particular and within the first part of the chapter, the conceptual aspects of individual context-aware environments will be discussed and the possible types of interactions will be outlined. The second part will discuss the potential for contextual reasoning and context prediction within such environments and will also provide a number of service categories for which a virtually unlimited number of tasks could be devised for and that would support various aspects of daily life activities.

\subsection{Context-Aware Computing}

The challenge for ubiquitous and pervasive computing is in managing a sophisticated and dynamic perspective on computer-mediated interactions with human beings without intruding unnecessarily into their lives. This is what is described as the notion of calm (Weiser 1991), where computing resources quietly modify themselves to suit the needs of the user. In order to make sense of ubiquitous computing environments, the word context has been used to describe how sensors, processors and actuators can interpret and influence the environment. As recognised in Dey (2001), the term context has been defined differently for different domains and moreover it may also mean different things to different people. The term contextaware computing has arisen from a well-established body of ubiquitous computing 
research concerned with location (Schilit et al. 1994). It was defined in the context of the systems in which the user employs many different mobile, embedded and stationary computers in different situations and locations over the course of the day. This has evolved from within several research fields sharing many common views, including ubiquitous computing (Weiser 1991; Dey and Abowd 1999), pervasive computing (Ark and Selker 1999) and ambient intelligence (Aarts and Collier 2003) where the term context refers to any information that can be used to characterise the situation of a person or a computational entity. Although, weak in a sense that it lacks a formal definition and instead includes practically all information, meaningful or not, in this chapter the latter definition is supported given the context of ambient intelligence. The reason for that is simply based on the fact that a given context can in fact be influenced by "any information". Thus, it is in fact the application that usually defines the scope of the context desired and not the environment. That is of course in dependence to the information that are available to the application. Note that depending on issues related to security, privacy, etc. some information available in the environment may be hidden from the application and as such cannot be used by it.

Research in human-computer interaction such as Dourish (2004) proposes a perspective on context-aware computing where the context is perceived much as in social sciences that study the practices of individuals in their normal environment. Here, the problem of context is examined from a high level, philosophical point of view, highlighting an approach that views context as an interactional problem rather than a representational one. Another form is that of dynamic contexts (Sterritt et al. 2005) where it is the activity that generates and sustains the context. So, context arises from the activity and is actively produced and maintained in the course of the activity. This provides a framework for a method to determine context from activity via behaviour (and measures of behaviour) and is in line with the research vision of Suchmann (1987). Indeed, the perspective of context arising from interactions is very close to the vision of research from those researching socially aware computing and communication, where social computing proposes ubiquitous computing environments and resources that understand social signalling and social context (Pentland 2005).

In order to manage context events, a framework is needed that supports the management of extensible context and context histories and makes it possible to utilise context in context-aware environments. However, it seems that there is no consensus for context or context history utilisation (capturing, representing, modelling and using context) over time. The development of research into context histories (Helander 2005; Wilson et al. 2005; Schneider et al. 2005) provides an important framework for higher level representation of labelled contexts and is a foundation for emerging research in such areas as the continuous archival and retrieval of personal experiences (e.g. Aizawa et al. 2004) as famously described in Bush (1945). Some practical problems include the fact that acquired information can be strongly heterogeneous and often incorrect, inconsistent or incomplete and, worse of all, not at all or insufficiently correlated with each other. A second issue is that context is used in systems in various ways which makes it difficult to understand if it is 
stored "out of context". A substantial number of different approaches have been proposed to model such contextual information. The most sophisticated propose a form of layered context structure framework, with a context provider layer for the incorporation of raw sensor and actuator data and a context service layer for context provision in context-aware environments (Huebscher and McCann 2005). This would allow for facilitating some form of computational model of context processing as in Balkenius and Moren (2000) and Strang and Linnhoff-Popien (2004) that orchestrates context stimuli and components into a single and coherent representation or places the interaction into a practical framework (DiDuca and Van-Helvert 2005). There is also a requirement to gauge the quality of our contextual information and histories objectively as it is gathered, as from the Quality of Context (QoC) mechanism of Buchholz and Kupper (2003), in which any contextual information comes associated with parameters including precision of information, correctness probability, trustworthiness, resolution and recency. Simply said, contextual information cannot be reduced to a trivial set of data to be accessed by components, but requires some higher form of organisation that correlates individual data entities with each other and more importantly with the application itself.

Overall, there is a very clear research roadmap for context-aware computing, driven in many ways by researchers who care a great deal about people and the interaction of people in independent living environments. Providing a true context stack is a significant research challenge and clearly the incorporation of a context reasoning and prediction layer into such a stack could both provide intelligent support and also facilitate strong communication capabilities via social inter-networks of family, friends, peers and others.

\subsection{Context Environments}

The term smart environment is usually used to refer to sensor-rich infrastructures that allow for the gathering of raw contextual data. The technology deployed within such environments varies from simple sensors over localised environments in order to detect, e.g. a door opening and closing to more elaborate sensor systems that reflect openly accessible smart infrastructures as necessary to achieve full contextual-awareness at various levels of granularity. The information therein may be sampled and monitored locally or remotely providing a framework where decisions can be made in instances of concern where, for instance, a person may require some form of intervention to, e.g. prevent a given situation worsening. In these environments, the technological sensing capability already allows not only to monitor the movements of a person but also their interaction with devices or other stakeholders within the environment. For example, turning the cooker on or off, turning a tap on or off or adjusting the temperature within the home. Such data, if correlated correctly, can then be sequenced into chains of events that can be used to, e.g. advise next actions to be done in order to perform certain tasks. For instance, fill the kettle with water before switching it on. Alternatively, if compared to another event 
pattern, they can be used to detect unwanted or even dangerous situation before they actually happen. For instance, switching on the oven before going to bed is potentially dangerous and most certainly unwanted. Here an alarm could be raised or the cooker could be switched off automatically via an associated actuator.

Naturally, if desired information cannot be sensed, correlated or reasoned for then they are by default not available for subsequent tasks. Similarly, if a system lacks in understanding of the available information then their usefulness is questionable as the quality cannot be determined. Finally, no single service can address all of the requirements and complexities required in a given domain. Thus, the awareness and utilisation of other services that are available in the same or other environments are important for the success of individual services. Therefore the three core aspects for the successful deployment of contextual services in smart infrastructures can be summarised as (a) the sensing capabilities of the system, (b) the understanding thereof and (c) the services available on the system.

Considering the above and the distributed nature of the underlying sensor-based architecture smart environments may be categorised as follows:

- Known Macro-environments: Such environments are limited in a sense that they have clearly defined boundaries with respect to their spatial dimensions and sensorial capabilities. They are known in a way that they are purpose built and as such the type of information and their relation to each other is known. Services deployed in such an environment may take direct advantage of the knowledge that is incorporated in the infrastructure and the standardised interfaces thereof. Probably the most relevant example for such an environment is that of a smart home.

- Unknown Macro-environments: Also limited in the same sense as above but may be unknown to the application, service or user that are interacting with the environment. A good example for such an environment would be public smart buildings, e.g. hospitals, schools and parks. Such environments are by default unknown to a potential user and also differ with respect to the sensor and service infrastructure. However, resources deployed would be clearly defined so that the environment could be used exploratory. Here an additional layer would be required that is capable of bridging the gap between the environment and the service that are trying to utilise it.

- Unknown Openenvironments: Are defined to have no spatial or sensor (or at least very open) boundaries but are equipped with smart devices that can be accessed by potential users and applications and on which other users can deploy their own services, e.g. the physical layout of a large city may represent such an environment, where spatial boundaries are practically not present and where a multitude of different sensors and services may be available from unknown and as such uncertified sources. In such an environment the mapping between the infrastructure and the services would be very difficult, if not impossible, if no degree of standardisation or contextual description exists.

- Specialised Micro-environments: In some cases, small scale and highly specialised environments may also be considered as contextual environments. For 

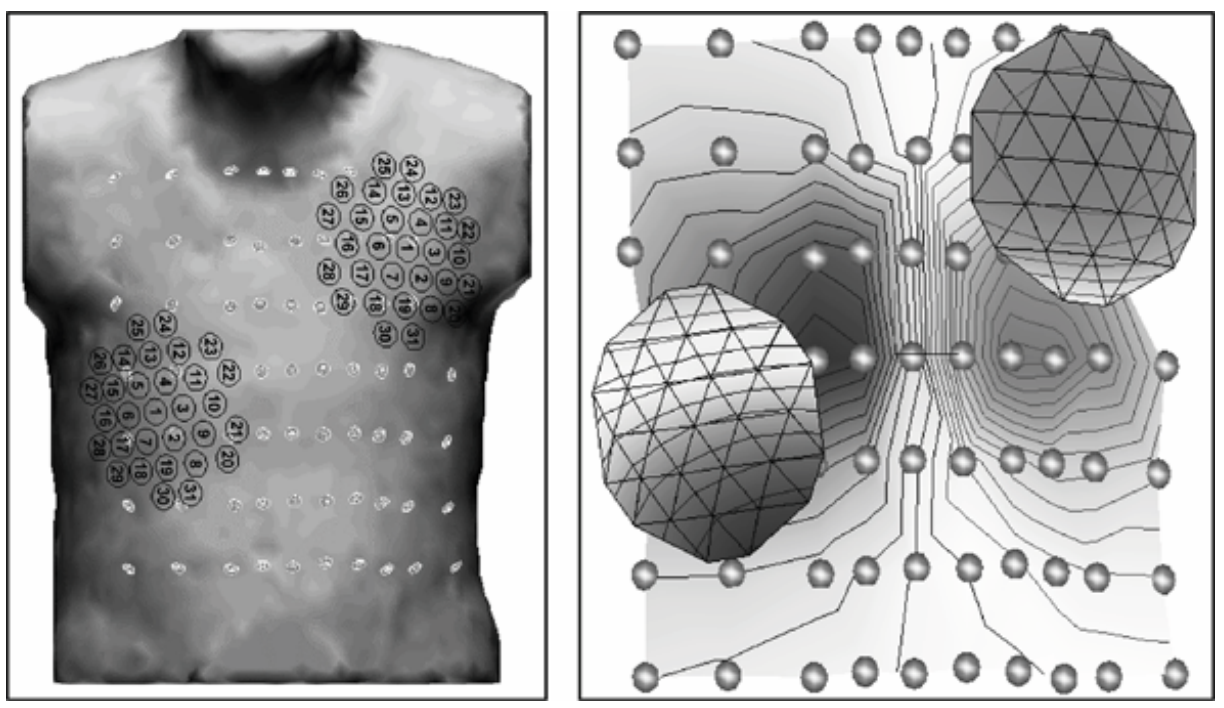

Fig. 9.1 Localised diagnostic environment (Emerald Insight 2009)

instance, Fig. 9.1 depicts a localised diagnostic environment applied to a person. If conditions require the detailed and perpetual monitoring of micro-aspects within a more globally orientated context environment then it would certainly be beneficial if such specialised micro-environments become part of the overall contextual infrastructure, e.g. within this example, a person's cardiac-related bio-signs are constantly measured and may be analysed locally. If connected to a smart home environment, for instance, a person's exercise activity could be performed and synchronised with their current physical condition as measured through the diagnostic system. This would avoid not only that a person is exercising too much but it could also advise to stop exercising if current heart condition would not warrant this.

\subsection{Context-Driven Interaction}

Similar to the above environment-based categorisation, services can be categorised based on the stakeholders involved or the type of interaction performed. In relation to the environment-based categorisation, the following three categories can be distinguished:

- People-to-Environment Interaction: Considering a localised smart environment as depicted in Fig. 9.2a, which illustrates a home environment. The flexible and dynamic interaction between each individual aspect of such an environment and its inhabitants, and vice versa, offers a wide range for possible pervasive services that could help facilitating daily life activities. Similarly, consider a more global-orientated smart world infrastructure such as depicted in Fig. 9.2b. Such 


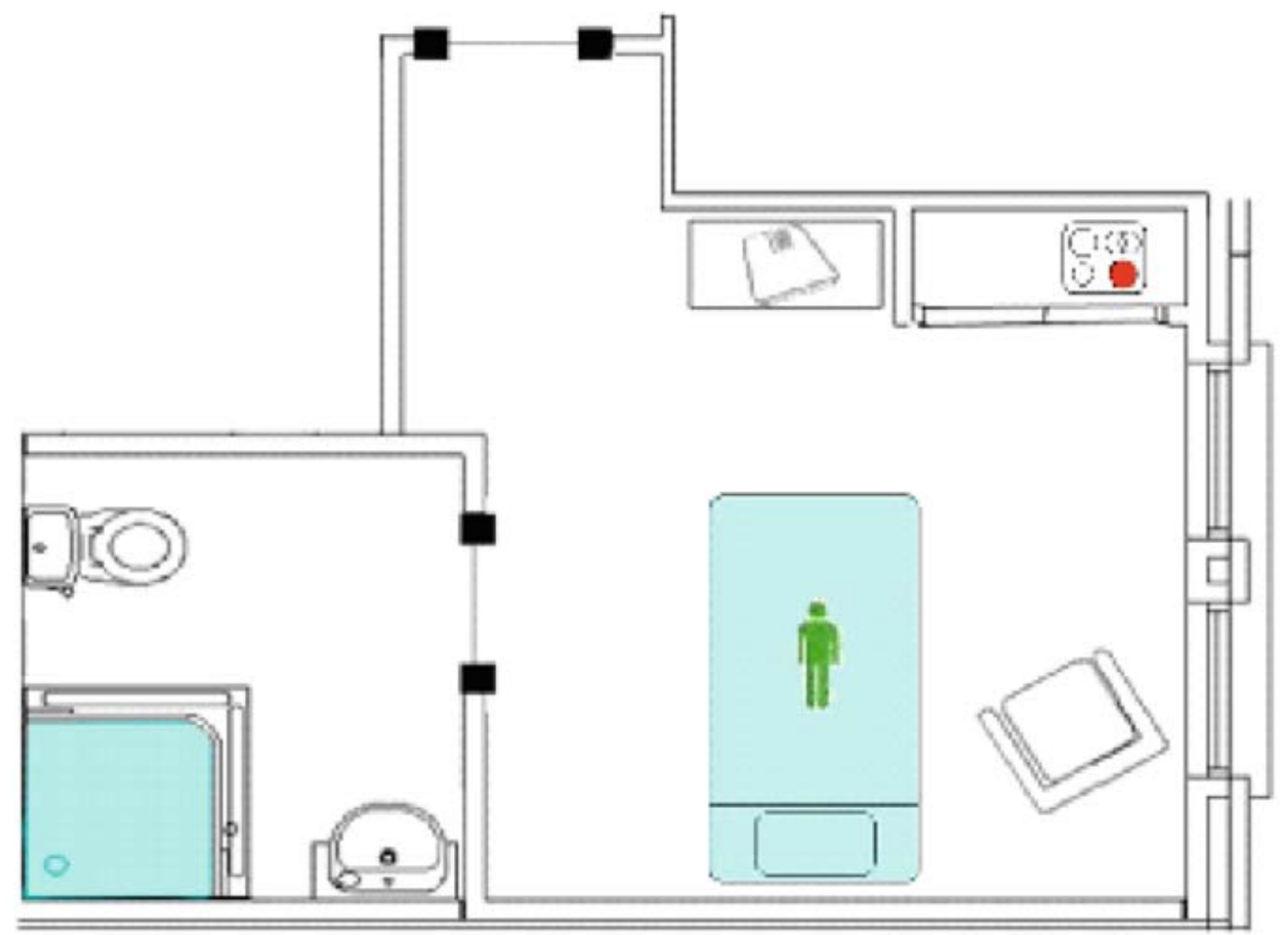

(a) Smart Ervironment

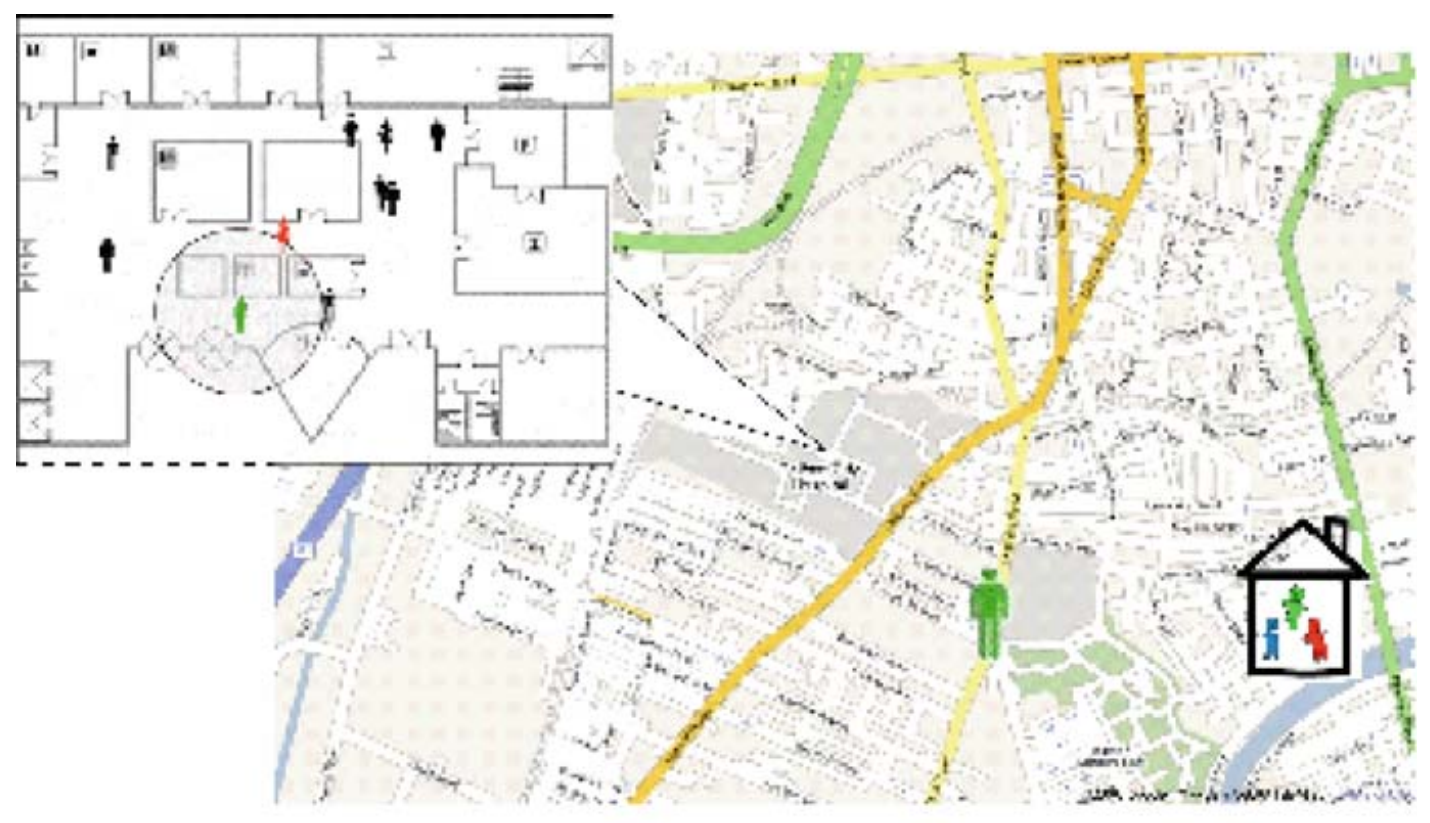

(b) S murt World Infm structure

Fig. 9.2 People-to-environment interaction 
an environment is by far more dynamic, that is, the type and amount of available contextual information are much more diverse and larger, respectively. Furthermore, the types of services to be applied may differ for different locations. For instance, the same type of service in two different towns may produce a different result. As for (a) potential services include the tracking of the person's movement within the house of the use of appliances and for (b) a kind of guideMeTo (Location(x)) service could be used to actually guide a person from a given location to another one, e.g. a hospital for weekly checkups. To ensure the safety of the patient this service would need to interact with localised services such as traffic lights.

- Environment-to-Environment Interaction: The interaction between environments themselves or, to be more precise, between the services deployed in them forms another important category of context-driven interaction. Such communication is not only relevant to interlink individual environments but also in instances of mobility, for instance, if a person moves from one environment to another. A "handover" can be initiated that transfers certain services or responsibilities that are relevant to this particular person to the destination environment. Another potentially useful application would be that similar environments could share their "expertise" in order to optimise their own services.

- People-to-People Interaction: The interaction between people represents another area where dedicated pervasive services can be applied. In this case specific interactions may be invoked; configured or individual personal interest may be shared to achieve separate goals. The identification of individual interaction entities and the negations of available and needed services represent distinct objectives in this area that would allow for the identification, localisation, spatial guidance and other person-to-person services.

\subsection{Towards Context Reasoning and Context Prediction}

Context-aware environments will undoubtedly play a vital role in the provision of next-generation person-centric and situation-specific services. However, they also provide the basis for advanced reasoning and prediction capabilities that may lead to intelligent environments that are intrinsically interwoven with services and as such would be able to offer a large degree of intelligent features as required for fully autonomic environments. In particular, such environments would be more flexible with respect to their use; they would be more resilient and failsafe and would in general be able to provide a higher degree of interaction as well as understanding. For instance, if a person detection sensor in a room would fail for any reason, the associated service could reason that "if the door has been opened and the light has been switched on" there is a person in the room. Vice versa, "if the light has been switched off, the door closed and nobody is lying in the bed" then the likelihood that nobody is in this room is high. Obviously, such reasoning is not always failsafe and as such needs to interact with as many information sources as possible to 
validate individual results from various scenarios. This is of particular interest if individual sensor information contradicts each other. For instance, a sensor embedded in a light switch indicates that the light is switched on. However, a separate light sensor in the same room registers the light to be switched off. Obviously, such a situation would indicate a fault in the senor environment and a reasoning engine could trigger corrective measures. Nevertheless, the question of the light being on or off still remains. In order to answer this question additional sensors would be required that either sense the same concept of the existing sensors, thus introducing a level of redundancy over which a system can reason, or alternatively a separate concept needs to be sensed, which needs, however, to be associated with the problem concerned. For the example discussed the power intake of the room could be measured and correlated with the devices activated in the room in order to determine if the light is actually on or off. In the literature, reasoning tasks can be categorised to be inductive, deductive or abductive. Deductive reasoning is a top-down approach that allows to (in-)validate a theory or condition. For instance, "the light is on" if "light switch = on" and "light sensor = bright". Inductive reasoning on the other hand is a bottom-up approach and seeks a broader generalisation that is based on specific observations. For example, if "light switch $=$ on" and "light sensor $=$ bright" then "the light is on". Finally, adductive reasoning attempts to infer logical explanations based on specific observation. For instance, if "light sensor = bright" then "light switch $=$ on". However, unlike the principles of deduction this statement does not necessarily infer the fact that "the light is on". In abduction the inferred results do not have to be necessarily true in the wider context of the problem that is under scrutiny. Instead they are possible explanations that may be further validated using deduction.

Taking the above into account reasoning can be used to (a) prove hypotheses that lead to generalised rules of behaviour, (b) infer an hypothesis based on observations which in turn can be further validated and (c) explain the dependencies between concepts. Now in order to answer the question of if the light is actually on or off, consider the scenario depicted in Fig. 9.3.

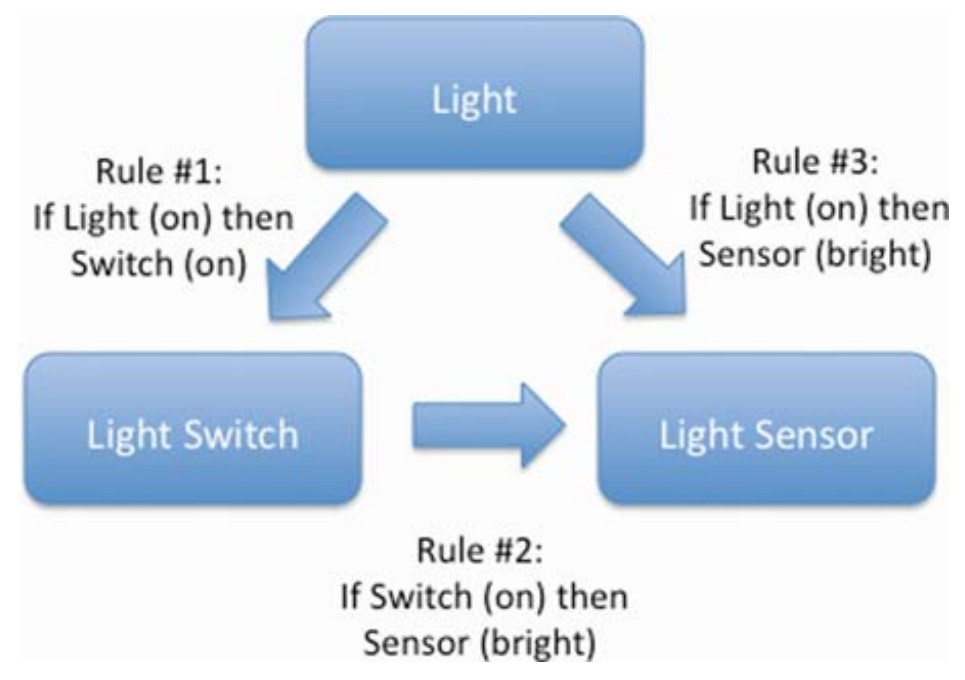

Fig. 9.3 Contextual reasoning 
Here the concept under scrutiny is if the light is on or off and the parameters under observation are a light switch and a light sensor. Through common sense two rules have been established stating that if the light is on then the switch is on and that if the switch is on the sensor is sensing bright. Note that Rule \#3 is not known beforehand. The goal is to verify that the light is actually on if the switch is set to on. For this consider the following: if the light switch is set to on then it can be, through inductive reasoning and Rule \#1, inferred that the light is on. However, at this stage this is only a hypothesis that is not yet proven. Rule \#2 states that the sensor is bright if the switch is on which infers, through abductive reasoning, that the sensor is also bright if the light is on. Thus, Rule \#3 can be generated. Now, through deductive reasoning it can be argued that the light is indeed on if the switch is set to on and if the sensor is sensing bright.

Although very simple, the above scenario illustrates the potential of the various reasoning mechanisms for context-aware environments. In particular the verification of individual contexts in relation to given parameters will depend heavily on such mechanisms.

Another important aspect for context-aware environments is that of context prediction, which in a context-aware environment makes possible proactive devices and device interfaces that go some way towards the provision of a calm environment, as envisaged by Weiser (1991). We define context prediction as the ability to predict the possible future contexts of interaction with people or other contextual environments, as described by Mayerhofer et al. (2003). This means that individual behaviourally based profiles may be accessed and used to understand and manage behaviour traits. However, this is a significant research challenge requiring dynamic, layered, socially orientated and extensible context "stacks" with new research required especially for the definition of a context prediction stack. DiDuca and Van-Helvert (2005) state that "service offerings may be based on such things as rhythms or patterns of behaviour, body language, etc. which suggests that we may have to look beyond mainstream approaches and consider methods from different domains such as ethnographic, observational or pattern recognition approaches." Some research perspectives on possible context architectures provide helpful insights. In particular, Nurmi et al. (2005) reason that context monitoring and reasoning require large amount of resources and to address this issue perhaps distributed and peer-to-peer approaches could be used. We have examined this issue in a related research area (Mulvenna and Zambonelli 2005) and believe that it does show promise, perhaps particularly, in the area of use of semantic overlay technologies as researched in Loeser (2003) and Nurmi et al. (2005) also raises the important issue of prediction sharing, where individuals or their environments may have access to the contexts, context histories and/or context predictions of others. In their research, Petzold et al. focus also on context prediction based on previous behaviour patterns. Their proposed prediction algorithms originate in branch prediction techniques (known from the area of processor architecture), which are transformed to handle context prediction (Petzold and Bagci 2003). Irrespective of the use of predictive mechanism in general it holds that the better the accuracy and the longer the prediction is valid for the more useful it will be for any form of guidance or system adaptation. 


\subsection{Context-Aware Computing in Support of People with Dementia}

With over 5 million people affected by dementia in all of Europe (Alzheimer's Europe 2009) it becomes clear that new and cost-effective ways are needed to provide the best care possible. Providing home support at various levels from an early stage onwards could avoid or at least delay institutionalisation which in turn would increase the quality of life of a patient while simultaneously being more costeffective. Within an intelligent context-aware environment, a number of different categories of services could be thought of for which virtually an unlimited number of individual services could be deployed that can be used to support a wide range of daily task activities. Figure 9.4 depicts some categories for which various services could be devised that actively reinforce a person's confidence for certain tasks, utilises reminders to guide certain activities, improves and maintains social interaction or helping caretakers and doctors by providing relevant support in relation to the person cared for.

In short, such services do not only have the potential just to increase the confidence and safety a person is experiencing but do have the potential to prolong independent living beyond current threshold. This is particularly relevant for dementia-related care where a person's physical fitness would often allow for an independent lifestyle but cognitive decline may necessitate institutionalisation. By providing relevant guidance and safety layers such that people could live independently for longer would ultimately improve quality of life. In order to realise such services realistically, a generalised framework is required that supports the management of extensible context and context histories and makes it possible to utilise context in ubiquitous environments in a flexible and open manner. However, as

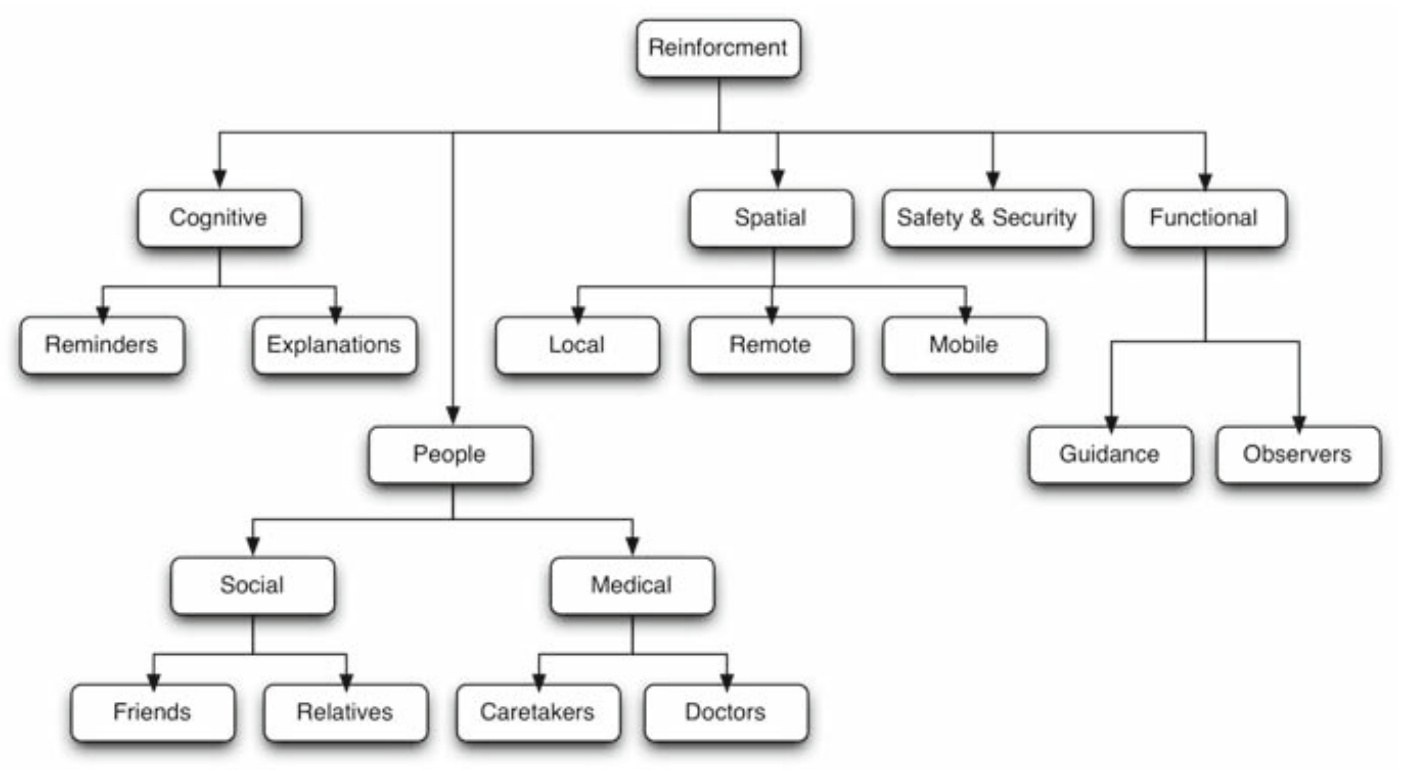

Fig. 9.4 Example of service types supporting daily life activities 
stated earlier, there is no general consensus yet for the use of context or context history-related information.

\subsection{Conclusion}

It has been shown that there is a clear need for intelligent services that are able to support ageing people in general but in particular those people suffering from certain medical conditions such as mild dementia but wish to continue to live independently. The types of services that could be offered in context-aware environments will centre on cognitive and social reinforcement in addition to the functional support for daily activities. Contemporary mobile devices such as personal digital assistants (PDA) or smart phones are already being specifically tailored towards the use by elderly and disabled people so that technology will be actually supporting rather than being an obstacle. The major benefit of such technology is that people can remain in their home environment for longer which will not only be more costeffective but will also improve the quality of life for the person and the family and friends around them. However, if such services are limited to the home itself then this could lead to the virtual imprisonment of people in their own home. The use of portable technology such as mobile phones can ensure the continuity of services beyond the limits of individual macro-environments. However, to be fully effective, contextual environments have to be made available in a fully pervasive and open fashion at all levels of granularity.

There are valuable lessons from socially orientated research in usability and situated action that has to be incorporated into intelligent context-aware environments for context-aware technology to be successful. This chapter has reviewed and examined layered and extensible context architectures that provide self-managed and self-configuration autonomic capabilities. In addition to the context provider and context service layers, context reasoning and context prediction layers with cross-layer QoC capabilities have been discussed.

\section{References}

Alzheimer's Europe. (2009) www.alzheimer-europe.org, last accessed February 2009.

Alzheimer's Organisation. (2009) www.alzheimers.org, last accessed February 2009.

Aarts, E., Collier, R. (2003) Ambient intelligence. First European Symposium, EUSAI 2003, Springer, Veldhoven, The Netherlands.

Aizawa, K., Tancharoen, D., Kawasaki, S., Yamasaki, T. (2004) Efficient retrieval of life log based on context and content. The First ACM Workshop on Continuous Archival and Retrieval of Personal Experiences (CARPE-2004), Columbia University, New York.

Ark, W., Selker, T. (1999) A look at human interaction with pervasive computers. IBM Systems Journal, 38(4):504-507.

Balkenius, C., Moren, J. (2000)A computational model of context processing. Sixth International Conference on the Simulation of Adaptive Behaviour, The MIT Press, Cambridge, MA.

Buchholz, T., Kupper, A. (2003) Quality of context: What it is and why we need it. Workshop of the HP OpenView University Association 2003 (HPOVUA 2003), Geneva.

Bush, V. (1945) As we may think. Atlantic Monthly, 176(1):101-108. 
Dey, A.K., Abowd, G.D. (1999) Towards a Better Understanding of Context and ContextAwareness, College of Computing, Georgia Institute of Technology, Atlanta GA, USA.

Anind, K.D. (2001) Understanding and using context. Personal and Ubiquitous Computing Journal, 5(1):pp. 4-7.

DiDuca, D., Van-Helvert, J. (2005) User experience of intelligent buildings: A user-centre research framework. IEEE International Workshop on Intelligent Environments, Colchester, UK.

Dourish, P. (2004) What we talk about when we talk about context. Personal and Ubiquitous Computing, 8(1):19-30.

Emerald Insight. (2009) http://www.emeraldinsight.com; last accessed February 2009.

Helal, S., Winkler, B., Lee, Ch., Kaddourah, Y., Ran, L., Giraldo, C., Mann, W. (2003) Enabling location-aware pervasive computing applications for the elderly. Proceedings of PerCom 2003 - 1st IEEE Conference on Pervasive Computing, Fort Worth, Texas.

Helander, J. (2005) Exploiting context histories in setting up an E-Home. 1st International Workshop on Exploiting Context Histories in Smart Environments (ECHISE-2005), Munich, Germany.

Huebscher, M.C., McCann., J.A. (2005) Adaptive middleware for context-aware applications in smart-homes. 2nd Workshop on Middleware for Pervasive and Ad-Hoc Computing (MPAC), Grenoble, France.

Loeser, A.F. (2003) Naumann: Semantic overlay clusters within super-peer networks. International Workshop on Databases, Information Systems and Peer-to-Peer Computing (DBISP2P), Berlin, Germany.

Mayrhofer, R., Radi, H., Ferscha, A. (2003) Recognizing and Predicting Context by Learning from User Behaviour, Austrian Computer Society (OCG).

Mulvenna, M.D., Zambonelli, F. (2005) Knowledge networks: The nervous system of an autonomic communication infrastructure. 2nd IFIP TC6 International Workshop on Autonomic Communication (WAC 2005), Vouliagmeni, Athens, Greece.

Nurmi, P., Martin, M., Flanagan, J.A. (2005) Enabling proactiveness through context prediction. Proceedings of the Workshop on Context Awareness for Proactive Systems, Helsinki.

Pentland, A. (2005) Socially aware computation and communication. Computer, 38(3): 33-40.

Petzold, J., Bagci, F. (2003) Global and local state context prediction. Artificial Intelligence in Mobile Systems 2003 (AIMS 2003) in Conjunction with the Fifth International Conference on Ubiquitous Computing, Seattle, USA.

Schilit, B.N., Adams, N., Gold, R., Tso, M.M., Want, R. (1994) Context-aware computing applications. IEEE Workshop on Mobile Computing Systems and Applications, Santa Cruz, California, USA.

Schneider, M., Bauer, M., Kröner, A. (2005) Building a personal memory for situated user support. 1st International Workshop on Exploiting Context Histories in Smart Environments (ECHISE2005), Munich, Germany.

Sterritt, R., Mulvenna, M.D., Lawrynowicz, A. (2005) Dynamic and contextualised behavioural knowledge in autonomic communications. First International IFIP Workshop WAC 2004, Springer-Verlag, Lecture Notes in Computing Science, Berlin.

Strang, T., Linnhoff-Popien, C. (2004) A context modeling survey. Workshop on Advanced Context Modelling, Reasoning and Management as Part of Ubiquities Computing - The Sixth International Conference on Ubiquitous Computing, Nottingham, England.

Suchman, L. (1987) Plans and Situated Actions: The Problem of Human-Machine Communication. Cambridge University Press, Cambridge, UK.

Weiser, M. (September 1991) The computer for the 21st century. Scientific American, 265(3): 94-104.

Wilson, D.H., Wyatt, D., Philipose, M. (2005) Using context history for data collection in the home. First International Workshop on Exploiting Context Histories in Smart Environments (ECHISE), Munich, Germany. 